\title{
Design Reuse Experience of Space and Hazardous Operations Robots
}

\author{
P. Graham O'Neil \\ Automation and Robotics Department \\ Lockheed Engineering and Sciences Company \\ 2400 NASA Road 1, Houston TX 77058 \\ oneil@ajo.jsc.nasa.gov \\ January 12, 1994
}

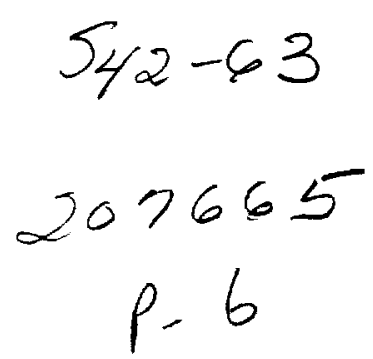

\begin{abstract}
A comparison of design drivers for space and hazardous nuclear waste operating robots details similarities and differences in operations, performance and environmental parameters for these critical environments. The similarities are exploited to provide low risk system components based on reuse principles and design knowledge. Risk reduction techniques are used for bridging areas of significant differences. As an example, risk reduction of a new sensor design for nuclear environment operations is employed to provide upgradeable replacement units in a reusable architecture for significantly higher levels of radiation.
\end{abstract}

\section{Introduction}

Robotics operations in hazardous environments are attractive because they reduce exposure and risk to humans, perform reliably in hostile environments, and can be used to amplify human capabilities. The environments receiving the most attention for these applications have been underwater, outer space or on earth in areas where radioactive or hazardous materials pose threats to humans and their automated equipment. These environments possess some common characteristics, yet each is distinct in its engineering design challenges. As technology growth presents more economic alternatives, this list of working environments will grow. This paper presents a framework for cataloging reuse features, assessing benefits of the transfer from one environment to another and emphasizes the decisions made early in the life cycle for optimal reuse.

Applications of design reuse and tailoring techniques for applications as diverse as laboratory automation of hazardous contaminants for the Contaminant Automation Analysis (CAA) Program to automated equipment used in hazardous waste tank operations, burial pit operations and mining extraction processes will be presented. An overview is presented in Table 1 for these applications and environments.
Typical design features driven by non-functional requirements that reuse knowledge or design details common to both space and hazardous waste operations equipment include the following:

- Safety

- Development risk reduction

- Manufacturing and production quality requirements

- Environment

- Human Machine Interface design for efficiency and safety

- Maintainability

- Reliability

A comparison of design features based on these require ments for laboratory analysis applications projected for environmental and space operations is given in Table 2.

A direct comparison of operational environments for the inspection tasks and light utility duty is given in Table 3 for the Special Purpose Dextrous Manipulator System being developed for the Space Station and the Light Duty Utility Arm for inspection tasks in the Hanford Single Shell Tanks.

\section{Common Design Goals}

For automated remediation operations in hazardous waste tanks, key operating parameters are driven by task/path planning and motion control. A high level view of the automation activities associated with task planning and execution of operators goals includes these activities:

- Direct robot to start task

Copyright c 1994 by Lockheed Engineering \& Sciences Co. Published by the American Institute of Aeronautics and 


\begin{tabular}{|l|l|l|l|}
\hline Application & Nuclear & Space & Underwater \\
\hline Lab Analysis & CAA & DART & - \\
Inspection & Hanford tanks & Space Station & Oil Rig and Cable Inspection \\
Soil Movement & INEL Pit 9 & Lunar regolith processor & Dredging and Harbor cleanup \\
Assembly & Fuel Reprocessing & SS maintenance, FLO prep & Pump and Pipe placement \\
\hline
\end{tabular}

Table 1: Application and Environment Analogies

\begin{tabular}{|l|l|l|}
\hline Design feature & DART/NASA & Contaminant Automation Analysis [DOE] \\
\hline Packaging & Must assure containment & Integrated and self-contained \\
Workspace & Standard Lab module & Up to 8 standard modules in series \\
User Interface & Virtual Operator/Telepresence & High level user interface \\
Remote operator latency & $>3$ seconds & $1-3$ seconds \\
Computer architecture & Real time UNIX & Real time Unix \\
Chemical Exposure & O, UV, high pH & acid fumes pH $\uparrow 14$ \\
Radiation [dose] & $2 \mathrm{Rad} / \mathrm{hr}$ & $250 \mathrm{Rad} / \mathrm{hr}$ \\
Radiation [lifetime] & $10^{5}$ & $10^{8}$ \\
\hline
\end{tabular}

Table 2: Laboratory Automation Comparison

- Search world model for access points

- Reason about tool selection for task

- Plan trajectory and workspace motion

- Present to operator for verification using the following interface channels:

- Remote Viewing

- Controller Inputs

- Shared Control Authority

- Graphics Display

- Audio Feedback

Adapting the first 4 activities for the unique features of the Hanford single shell tank waste remediation system, the following extensions for Force Controlled Interactions with the environment include are developed:

- Minimize normal force on walls

- Surface tracking of solid waste

- Threshold force application for breaking salt formations and selection of appropriate tool for autonomous grinding or sucking tasks.

- Oscillation compensation for dextrous tool application

Similar tasks involving force controlled interactions with worksite and environment exist on planetary surfaces for resource utilization and in the underwater environment for harbor dredging, or oil well infrastructure development.

\section{Reuse Processes}

This section presents details on the use of scenario analysis and testbed development and utilization to enhance the reuse process. The following section presents details on the role of design knowledge transfer, interface specification, and trade study reuse.

\section{Scenario Analysis}

The scenarios analysis is a tool used for requirements development and analysis. It provides a framework for multi-discipline teams to describe events and flows within the system and perform contingency modeling and analysis. The process can be applied to an conceptual and architectural model to investigate requirements defects. Scenario analysis has been used in the following manner.

Figure 1 is a scenario outline used for deriving advanced development requirements for maintenance robotics for the First Lunar Outpost (FLO). When similar approaches were applied to buried waste retrieval tasks, the result included better definition of tether monitoring and management tasks, and the inclusion of trades for periodic decontamination and maintenance actions.

\section{Simulation and Testbeds}

Design processes for implementing these functions for robots operating in a variety of environments [undersea, in the field, or in space], can use similar simulations, and analysis tools, further increasing the potential for reuse of robotic design knowledge to field reliable systems with greatest design maturity and least development risk. Examples of reuse of these simulations and testbed facilities are: 


\begin{tabular}{|l|l|l|}
\hline Parameter & Space [SPDM] & Radioactive Waste [LDUA] \\
\hline Temperature Range & -150 to $+150 \mathrm{~F}$ & {$[-20$ to $150 \mathrm{~F}]$} \\
Pressure Range & 0 to 29.92 & standard $+/-10$ in Water \\
Particulate & Micrometeoroid & severe dust st \\
Chemical Exposure & O, UV & acid fumes $\mathrm{pH} \uparrow 14$ \\
Radiation [dose] & $250 \mathrm{Rad} / \mathrm{Sec}$ & $2,000 \mathrm{Rad} / \mathrm{hr}$ \\
Radiation [tota]] & $10^{\mathrm{s}}$ & $10^{8}$ \\
Landing/Wind Loading & $4.3 \mathrm{~g}$ & $3.5 \mathrm{~g}$ at $120 \mathrm{mph}$ gust \\
\hline
\end{tabular}

Table 3: Operating Environment Characterization

1. Testbed facilities being developed at JSC have capabilities with broad application for other areas. Some of these features included in the testbed harnesses are remote operations, virtual reality interfaces, variable time delay loops, and coordinated multi-arm controllers. Other engineering test capabilities include instrumented dynamics testing facilities with useful payloads into the thousands of pounds.

2. Simulations of space environments can be developed form Earth based analogs if design features were embedded during development. For instance, one heavy equipment company has an analytic simulation of soil blade interactions with user specified inputs for soil characteristics and reduced values of gravity that would be suitable for lunar or Mars resource utilization advanced development studies.

3. Libraries of graphic kinematics for a wide range of robots, worksites, and operating environments are becoming available and with maturing engineering management direction should be critical in shortening the design cycle, minimizing design and schedule risk, and provide early access to the user community.

\section{Reuse Strategies}

This section presents an approach to applying the software engineering concept of Abstract Interface Specifcation to engineering design reuse and presents 2 examples of design knowledge transfer from diverse robotics fields to a design for inspection for the Hanford Single Shell Tanks.

\section{Interface Specification}

Based on experiences at software reuse, modifications were made to the approach that seemed the easiest to automate, the Abstract Interface Specification (AIS). The modifications included specification of technology maturity, remaining areas of risk, physical descriptions and resource requirements and reuse history.

This approach is also used in generating simulations based on reusable components. Briefly the AIS approach consists of specifying the required and provided Bervices for each component and information about state constraints and exception conditions. Since files constructed with these attributes can be browsed with reuse software, the effort was minimal to setup and use. Figure 2 is an example of the extended abstract interface specification entries for this approach.

Entries in the specification are augmented by physical descriptions and operations notes with resource budgets where required. In particular slots are assigned for design knowledge and application reuse history of the following items:

- Materials

- software

- persistent design objects

- standard mechanical components

- standard trades

Once a library of specifications in this format is established, domain engineers can query or browse for suitability and closeness of application using a Knowledge Dictionary System approach. Establishing this library is one of the critical items in developing an engineering reuse process. This library and its tools for browsing provide the ability to use Commercial Off-The-Shelf (COTS) components with confidence, design robust systems with mature deissgn techniques, and include details for unique requirments based on modifications of closely approximated configuration items.

\section{Design Knowledge Transfer}


The use of atandard trades studies and design knowledge transfer is illustrated by the following 2 case studies for the Hanford single shell tank Light Duty Utility Arm (LDUA) inspection system. Characterization of deaign features in general terms with parametrization for different operations environments.

1. A vertical poeitioning mast for contamination containment and housing of a robotic inspection arm is necessary for the Hanford single shell tank inspection task. Given the geometries of the specified delivery system, a multi-jointed mast is required to meet the volume and length specifications. Figure 3 shows the trade variables that were examined and the evaluation of the engineering team for each of the three major concepts. Each of the 5 evaluation variables,

(a) Stifiness

(b) Smooth external surface

(c) Mast wall thickness

(d) Actuation

(e) Binge design

were chosen based on their contribution to top level requirements. Stiffness was derived from position accuracy requirements, smooth surface from contamination control and sealing requirements, and mast wall thickness from gross weight requirements. Design knowledge from experience in emplacement of masts for oil well drilling, marine structures, and simulation of multi-segment Space Station Remote Manipulator System (SSRMS) boom assembly was used to complete the figures of merit for positive contribution and relative importance weightings.

2. The selection [shown in Figure 4] of a mast position sensor component was driven by requirements for position accuracy, robustness in field operations and cost. Characterization of the 4 design choices,
(a) Mast markings
(b) Embedded Hall effect sensors
(c) Vertical position Sensors
(d) Laser ranging

was undertaken based on knowledge gained in automated factories, marine labs, government reports from DOE and NASA, and experience in precise position sensing for underground nuclear test monitoring. After risk reduction considerations were introduced to the selection process, an off the shelf laser ranging system using time of flight principles that had previously been integrated with a Proportional-Integral-Differential (PID) controller for boom management was seleçted.
This approach to trade studies and design knowledge transfer is well suited to projects with multi-discipline teams, tools for performing multi-attribute utility analysis and in need of a consistent basis for establishing design criteria and evaluation of alternate design choices.

\section{Conclusion}

Re-usability of design and knowledge from one environmental area to another is aided by use of object oriented technology approaches, scenario analysis, credible simulations, design knowledge libraries, and various classes of reuse tools. However caution is required since powerful tools require care and experience in their use on projects with mission and safety critical aspects. Emplacing the infrastructure to support this approach is beat if supported up front by management. 
Name:

Life Cycle Phase:

Type:

Purpose:

1. The scenario begins with the rover assigned to a mainterance action.

2. The manipulator element mates with the rover base.

9. Under teleoperated control, the combined rover moves to a point providing access for the best video of a failed system or SRU.

4. A maintenance engineer controls the combined arm and video system during the inspection task to diagnose the failure and plan for most effective repair.

5. Once a repair plan is generated, the mobile base with its attached arm moves to the commanded location of the spares supply.

6. The arm is used to load the required replacement $S R U$ s on the base according to access requirements for the planned maintenance actions.

7. The base with arm and payload navigates to the appropriate point to start system repair action.

8. The arm controlled by the teleoperator with inputs from video and supporting analysis to 10cate the failed module.

9. It removes the module and returns it to the equipment carrier on the base.

10. The arm locates the replacement $S R U$ and places it in the operational configuration.

11. After all maintenance and inspection actions are completed, the base traverses to the failed module crib [or equipment airlock].

12. It removes the failed items from the carrier and places them in the appropriate location.

19. When demate of the manipulator arm and its systems from the mobile base is complete, the scenario ends.

Figure 1: Lunar Outpost Maintenance Scenario

Risk Characterization:

Contexts:

With:

Note:

Rationale:

Provided Interface:

Resources:

Name:

Constraints:

Exceptions:

Notes:

Rationale:

Services:

Name:

Constraints:

Exceptions:

Notes:

Rationale:

Required Interfaces:

Resources:

Name:

Constraints:

Exceptions:

Notes:

Rationale:

Services:

Name:

Constraints:

Exceptions:

Notes:

Rationale:

State Constraints: ...

Figure 2: Structured Specification for Design Reuse 5 Browsing 


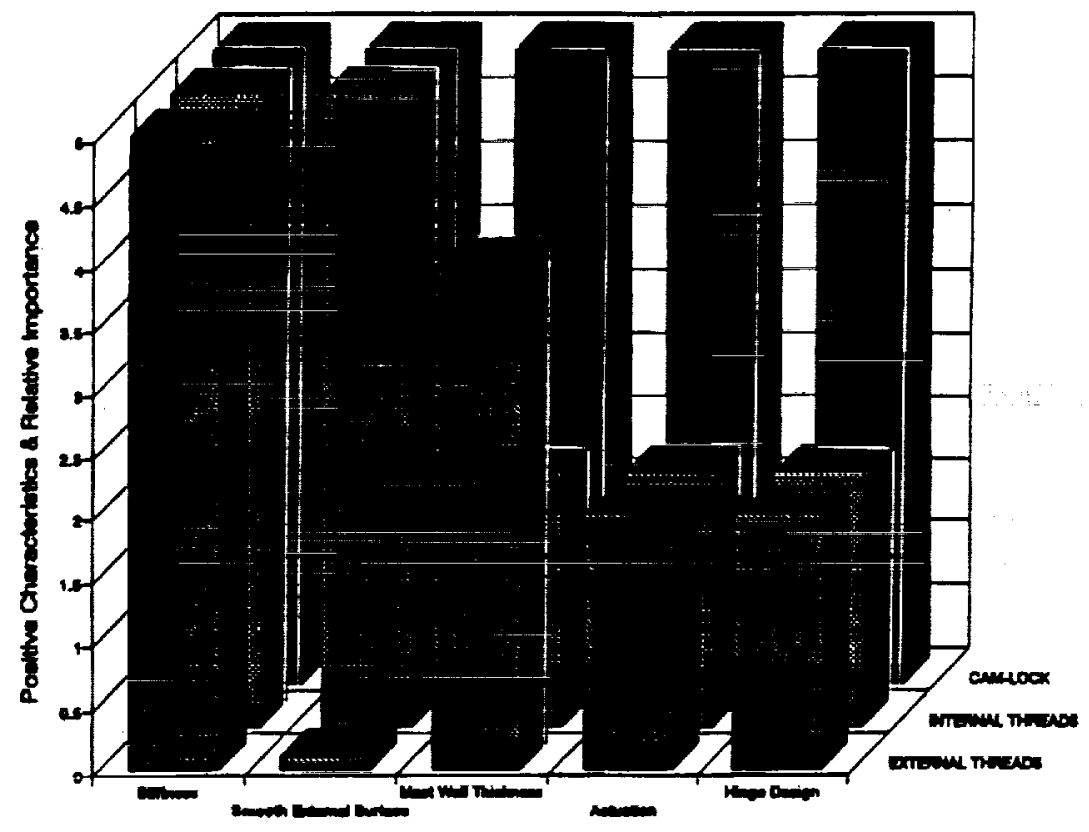

Figure 3: CAM LOCK Trades

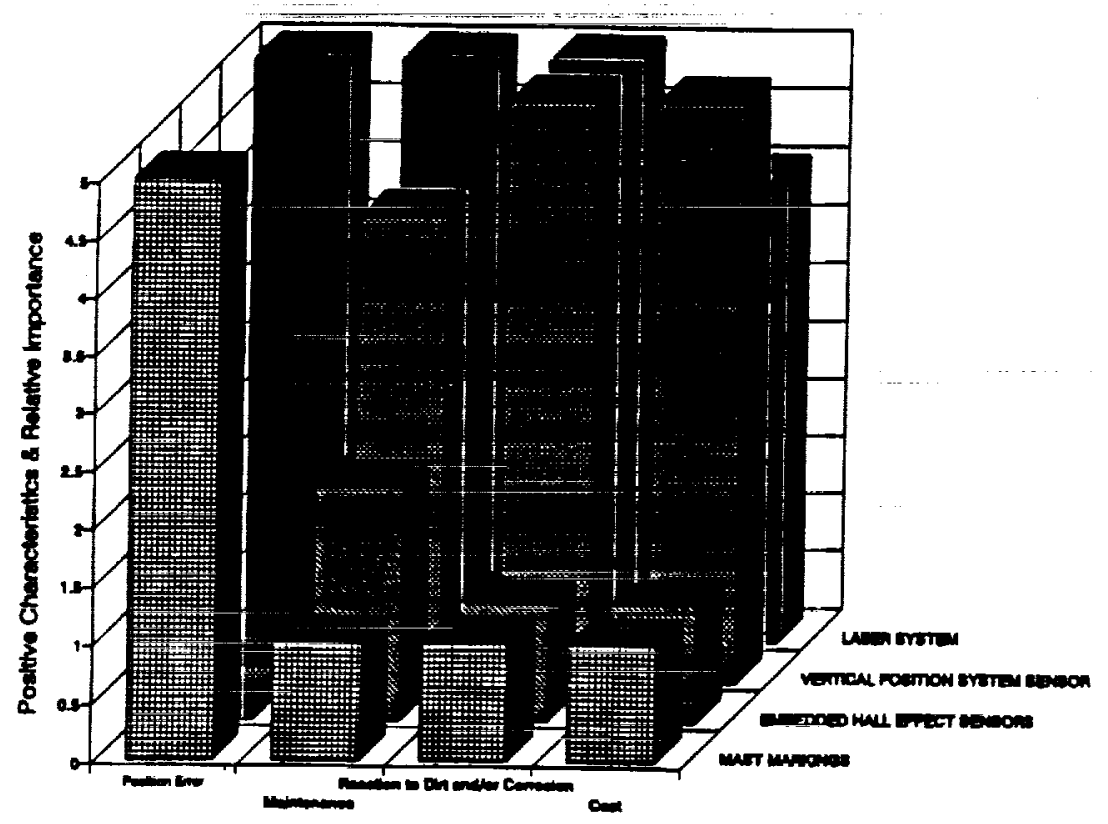

Figure 4: Mast Position Sensor Trades 